Mitochondrial encephalopathies with the mutation of the mitochondrial tRNA ${ }^{\text {Leu(UUR) }}$

Inui $\mathrm{K}$, Fukushima $\mathrm{H}$, Tsukamoto $\mathrm{H}$, et al. f Pediatr 1992;120:62-6.

The authors of this paper present clinical and laboratory data on four families with the common mutation of mitochondrial tRNA ${ }^{\text {Leu(UUR). }}$. In three of the families the index cases showed the typical presentation of mitochondrial myopathy, encephalopathy, lactic acidosis, and stroke-like episodes (MELAS). In the fourth family the proband did not have the stroke-like episodes. Two of the families also had first degree matrilineal relatives with unusual neurological and/or psychological symptoms. The mutation was identified initially in the probands using DNA from peripheral blood and muscle biopsy. DNA was then amplified by PCR using primers from mitochondrial nucleotide No 3130-3149 and 4262-4242 with subsequent digestion of the product with $A p a I$ restriction enzyme to yield a normal $1.1 \mathrm{~kb}$ and a mutant $1.0 \mathrm{~kb}$ fragment. An estimation of the degree of heteroplasmy was then attempted using Southern blotting. In three of the probands there was a higher proportion of mutant mitochondria in the muscle than blood; in the remaining case the proportion in each tissue was equal. Interestingly, the first degree relatives with unusual symptoms mentioned above also had detectable heteroplasmy; indeed, one sib with mental retardation, short stature, and diabetes had an indentical degree of heteroplasmy in both blood and muscle to her sister with MELAS. This study suggests that the phenotype associated with this mutation may be very variable.

\section{DAVID FITZPATRICK}

Fatal familial insomnia, a prion disease with a mutation at codon 178 of the prion protein gene Medori R, Tritschler H-J, LeBlanc A, et al. N Engl f Med 1992;326:444-9.

This paper describes a family affected by a disease characterised clinically by progressive insomnia, dysautonomia, and motor signs, and pathologically by selective atrophy of the anterior ventral and mediodorsal thalamic nuclei. In one patient spongiform changes in the cerebral cortex were found, suggesting that this condition, given the arresting title 'fatal familial insomnia' (FFI), might be a prion protein ( $P r P)$ disease. To investigate this possibility antibodies to $\operatorname{PrP}$ were used to perform dot and western blot analyses, with and without proteinase $K$, on necropsy brain tissue from two subjects with FFI. Protease resistant $\operatorname{PrP}$ was found in both. The coding region of the PrP gene was amplified and sequenced. Restriction enzyme analysis was then carried out with amplified PrP DNA from 33 members of the family. In the FFI family, four affected members and 11 of 29 unaffected subjects had a point mutation at $\operatorname{PrP}$ codon $178 \mathrm{GAC} \rightarrow \mathrm{AAC}$ resulting in substitution of asparagine for aspartic acid and elimination of a Tth111 I restriction site. Linkage was established between the point of mutation and FFI with $Z_{\text {max }}=3.4$ at $\theta=0$. FFI appears to be yet another prion protein disease, but differs from the phenotype seen in other families with the same point mutation. Genetic difference in post-translational conformational modification is only one of many possible explanations.

A M NORMAN

Detection of full fragile $X$ mutation

Pergolizzi RG, Erster SH, Goonewardena P, Brown TW. Lancet 1992;339:271-2.

This communication serves a dual function and has important implications for clinicians. Primarily the authors claim to have successfully developed a PCR method to identify the unstable genomic sequence in fragile X mental retardation syndrome. Such an encouraging development should make the application of diagnostic tests for this condition more widely available. Enthusiasm for the clinical application of these advances is tempered by the formidable technical difficulties which predictive testing for fragile $\mathrm{X}$ mental retardation may pose, as exemplified by the illustrative example which the authors provide. Prudently, the authors conclude that prediction in regard of affected females may be complex and recommend confirmation of PCR results by Southern blot analysis. In any situations caution must be the byword in the introduction of new technology to clinical practice if disservice to patients is to be minimised. While these tests will clearly interest members of fragile $\mathrm{X}$ mental retardation families and may even serve a beneficial purpose for some, this correspondent is unconvinced what benefits, other than commercial, may result from offering all women PCR based screening to determine their carrier status for the mutation in question, as envisaged by the authors of this report.

\section{WILLIE REARDON}

Expression of members of the putative olfactory receptor gene family in mammalian germ cells

Parmentier M, Libert F, Scheuermans S, et al. Nature 1992;355:453-55.

Of all the senses, that of smell has long been physiologically the most mysterious. The recent identification of large batteries (over
200) of G protein coupled olfactory receptor genes has changed this, suggesting that the diversity of olfactory sensation may be explained by the combinatorial excitation of different receptor repertoires. Parmentier et al add a new twist by showing that other members of the same receptor family are expressed in testicular tissue, including spermatocytes and spermatids. They make the intriguing suggestion that these receptors might be involved in sperm chemotaxis; study of the sea urchin provides some supporting evidence. Sperm expressing different receptors might therefore differ in 'virility': by coincidence, another article in the same issue of Nature provides evidence that such 'sperm competition' occurs in adders. At a practical level, further characterisation of these $\mathrm{G}$ protein coupled receptors could lead to new methods of contraception and infertility treatment.

ANDREW WILKIE

In vivo transfer of the human cystic fibrosis transmembrane conductance regulator gene to the airway epithelium

Rosenfield MA, Yoshmura K, Trapnell BL, et al. Cell 1991;68:143-55.

This paper describes the transfer of the human cystic fibrosis transmembrane conductance regulator (CFTR) cDNA to the airway epithelium of the cotton rats using a replication-deficient recombinant adenovirus vector (Ad-CFTR). The vector containing the CFTR cDNA was evaluated in vitro and showed expression of human CFTR mRNA and protein and correction of defective cAMP mediated $\mathrm{Cl}^{-}$permeability in $\mathrm{CF}$ epithelial cells. Following intratracheal administration of Ad-CFTR, human CFTR mRNA transcripts were detected in the cotton rat bronchial epithelium after two days by in situ hybridisation. Northern analysis showed persistent expression for up to six weeks. However, the level of expression was reduced to $42 \%$ of that observed at day 2 after infection. Immunohistochemical evaluation using antihuman CFTR antibody showed the presence of human CFTR protein in the bronchial epithelium 11 to 14 days after infection. These findings are an important step in the development of somatic gene therapy for cystic fibrosis since the lethal consequences of mutations of the gene involve the respiratory epithelium. However, questions about the safety (particularly in relation to acquisition of replication function by the replication deficient adenoviral vector) and the effectiveness (in relation to the regulation and level of expression of CFTR gene and specific respiratory epithelial cell targets necessary to reverse the disease process) remain to be answered. 